\title{
Characterization of coupling of pump fluctuations to laser in mode-locked Yb-doped and Er-doped fiber oscillators
}

\author{
I. L. Budunoğlu, K. Gürel and F. Ö. Ilday \\ Department of Physics, Bilkent University, TR-06800 Ankara, Turkey
}

\begin{abstract}
Transfer of fluctuations of pump power to laser power is characterized for mode-locked fiber oscillators. Contribution of pump noise to laser noise is estimated. Limits to pump modulation bandwidth for carrier-envelopephase stabilization are briefly discussed.

(C)2010 Optical Society of America

OCIS codes: (320.0320) Ultrafast optics; (060.2320) Fiber optics amplifiers and oscillators; (060.3510) Lasers, fiber; (060.7140)

Ultrafast processes in fibers; (140.3538) Lasers pulsed; (320.7090) Ultrafast Lasers
\end{abstract}

Mode-locked fiber laser oscillators are intensely studied in recent years. The most common applications include generation of optical frequency combs [1] and seeding of fiber, solid-state or parametric amplifiers. Virtually for all applications and experiments, but particularly for optical frequency metrology and amplifier seeding, power fluctuations of the laser should be minimized. Recently, we have reported a systematic characterization of intensity noise of mode-locked fiber oscillators operating in different mode-locking regimes and over a range of laser parameters [2]. The noise of these lasers, although typically quite low $(<0.1 \%)$, has significant contributions from technical noise, i.e., noise coupled from the environment or from the pump source. Since these can be eliminated with sufficient care, it is important to identify them. There is no systematic study, to our knowledge, of how fluctuations in pump power are transferred to laser noise.

Here, we investigate how variations in pump power are coupled to the mode-locked laser power as a function of frequency and over a range of laser parameters. A modulation or noise transfer function (referred to as NTF from this point on) is experimentally measured. From this information, we can roughly estimate how much of the laser noise is stemming from the pump source, which turns out to be substantial. In addition, these transfer characteristics is important for intentional modulation of pump power, which is the standard technique for locking the carrier-envelope phase (CEP) in fiber-based frequency combs [1]. The mode-locked fiber laser is, of course, not a linear device. Therefore, a transfer function cannot describe its dynamics fully. Recognition of this fact, on a different note, motivates us to view measurements of NTF under different laser operation modes an informative probe into the laser dynamics especially under unusual operation modes. As an example, the NTF measurements are made for a laser in its normal operation as well as in a high-noise state that the laser we have recently identified [2].

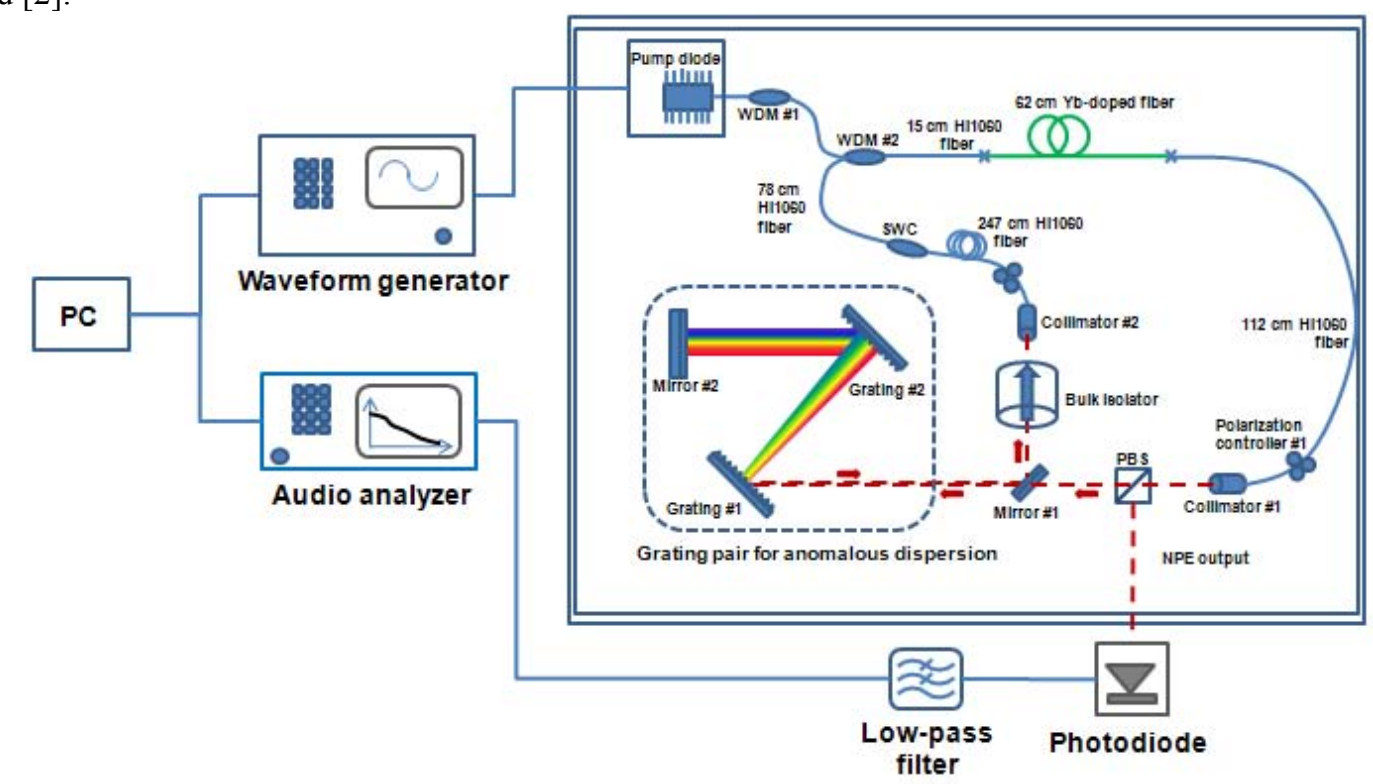

Fig. 1. Simplified diagram of the measurement setup.

An Yb-doped fiber laser (YDFL) and Er-doped fiber laser (EDFL) are used as test lasers. Mode-locking of both lasers is achieved by nonlinear polarization evolution. The YDFL operates in the similariton regime and EDFL operates in soliton-similariton regime, though the particular mode-locking regime is quite unlikely to alter the main results discussed here. The lasers are pumped in-core by a 980-nm pump diode with maximum $650 \mathrm{~mW}$ of power. The pulse repetition rate of the YDFL (EDLF) is $35 \mathrm{MHz}(40 \mathrm{MHz})$. 
We measure the intensity noise of the lasers with a high-dynamic range and low-noise baseband signal analyzer (Rohde \& Schwarz, Audio Analyzer UPV), following photodetection with a free-space InGaAs detector. In order to measure the coupling pump fluctuations to laser, we modulate the pump diode with an arbitrary waveform generator over a frequency range from DC to $250 \mathrm{kHz}$. The modulation amplitude is set to $\sim 0.03 \%$ to minimize nonlinear effects. We find that the NTF values change only slightly $(\sim 10 \%)$, when the modulation amplitude is doubled, suggesting weakly nonlinear response of the laser under normal operating conditions. The NTF is obtained via the same method as in [3].
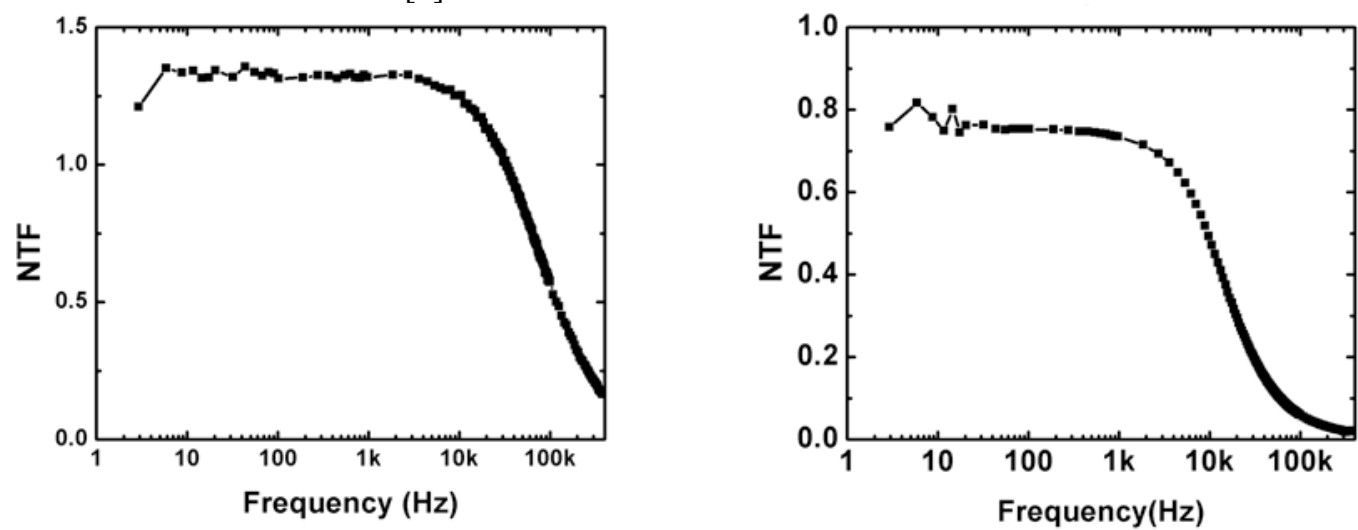

Fig. 2. Measured NTF for (a) a mode-locked Yb-doped fiber laser and (b) a mode-locked Er-doped fiber laser. The maximum modulation bandwidth is smaller for Er-fiber laser, as expected from their relative gain lifetimes.

The NTF measurements for the YDFL and EDFL are shown in Fig. 2. As expected, the NTFs are essentially flat up to a $3 \mathrm{~dB}$ cut-off frequency of $83 \mathrm{kHz}$ for the YDFL and $15 \mathrm{kHz}$ for the EDFL. The lower cut-off frequency is to be expected for Er-fiber, as the gain lifetime is longer than Yb-fibers by about the same amount. Using the NTF and pump laser spectral noise density (SND), the contribution to laser noise is calculated and compared to the measured values (Fig. 3 (a)). This suggests that the laser noise at low frequencies is dominated by noise transferred from the pump source. At frequencies above $\sim 10 \mathrm{kHz}$, the laser is above the level predicted by pump noise alone.
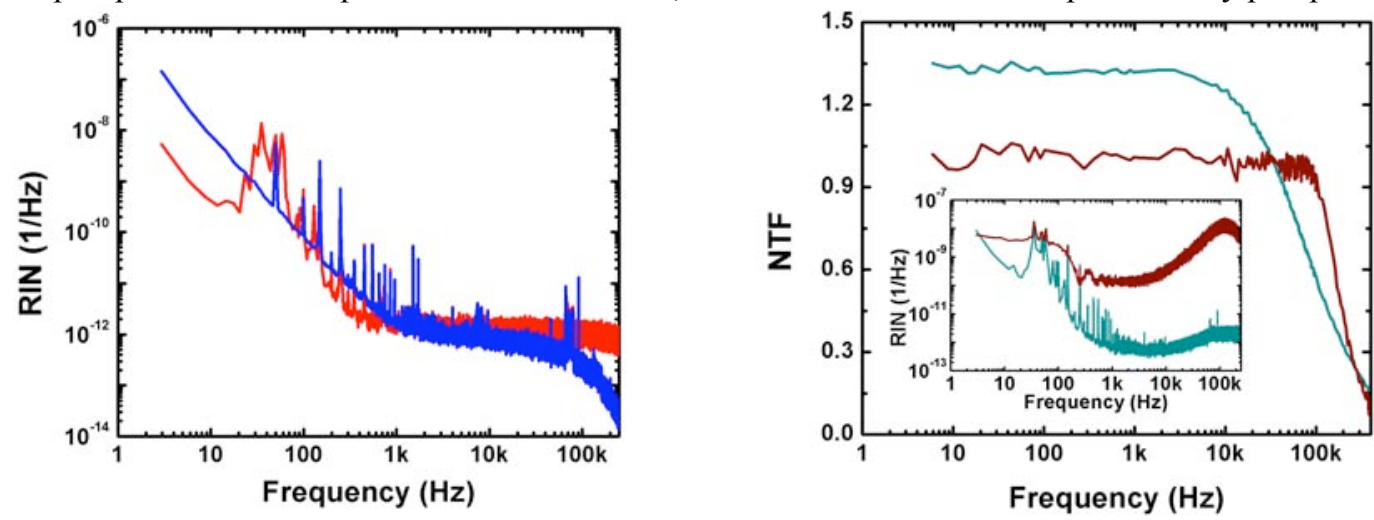

Fig. 3. (a) Measured (red) and predicted (blue) spectral noise density (SND) of the YDFL. (b) Measured SND and NTF of the YDFL for both the low-noise state and the ultrahigh-noise state. The differences in NTF are much less than the difference in noise.

Previously, we identified that the laser may switch to an ultra-high noise state at high pulse energies [2]. Next, we use the NTF measurement as a probe into this phenomenon. The NTF is measured both for the normal operational state of the laser (low noise) and also during the ultra-high noise state (Fig. 3 (b)). The fact that the NTFs differ proves that the response of the laser is more nonlinear, but the variation in the NTF (an increase of $18 \%$ ) is not nearly as much as the increase (by 58 times) in noise (inset of Fig. 3(b)). Thus, we conjecture that the dramatic increase in noise cannot be explained by gain dynamics alone.

In conclusion, we have measured the NTF of both Yb- and Er-doped fiber oscillators. We find that during normal operation, the response of the laser is only weakly linear. Pump noise appears to dominate laser noise at lower frequencies, which can be suppressed with proper feedback. The cut-off bandwidths for pump modulation are measured to be $83 \mathrm{kHz}$ for the YDFL and $15 \mathrm{kHz}$ for the EDFL, which is important for CEP stabilization for frequency combs. Finally, we use NTF measurements as a probe into the laser dynamics when its response becomes strongly nonlinear.

[1] N. R. Newbury, and W. C. Swann, "Low-noise fiber-laser frequency combs (Invited)," JOSA B 24, 1756 (2007).

[2] İ. L. Budunoğlu , C. Ülgüdür, Bulent Oktem, and F. Ö. Ilday, "Intensity noise of mode-locked fiber lasers," Opt. Lett. 34, 2516 (2009).

[3] R. P. Scott, C. Langrock, and B. H. Kolner, "Amplitude and envelope phase noise of a modelocked laser predicted from its noise transfer function and the pump noise power spectrum," IEEE J. Quantum Electron. 7, 641 (2001). 